\title{
Clinical and computed tomography imaging features of renal medullary carcinoma: A report of six cases
}

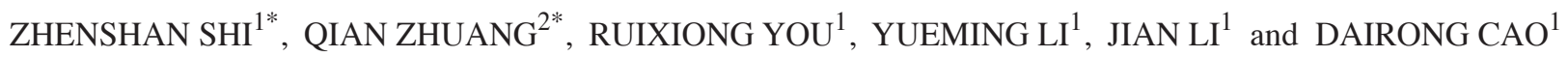 \\ ${ }^{1}$ Department of Radiology, First Affiliated Hospital of Fujian Medical University, Fuzhou, Fujian 350005; \\ ${ }^{2}$ Department of Pharmacy, Union Hospital of Fujian Medical University, Fuzhou, Fujian 350001, P.R. China
}

Received January 10, 2015; Accepted August 6, 2015

DOI: 10.3892/ol.2015.3891

\begin{abstract}
Patients with renal medullary carcinoma (RMC) have a poor prognosis, usually due to late diagnosis. Computed tomography (CT) analysis may aid the differentiation between RMC and other types of renal cell carcinoma, in order to establish an accurate early diagnosis. There is a limited number of reports in the literature focusing on clinical and multi-slice CT (MSCT) imaging findings of RMC. Consequently, the present study aimed to characterize the clinical and MSCT imaging features of RMC. For this purpose, the MSCT imaging findings of 6 patients with RMC were retrospectively studied. The patients were subjected to MSCT in order to investigate the characteristics of the tumors, including location, size, density, calcification, cystic or solid appearance, capsule sign, enhancement pattern and presence of retroperitoneal lymph node metastasis. The tumors in the current study presented a mean diameter of $7.48 \pm 3.25 \mathrm{~cm}$, and were observed to be solitary and heterogeneous with necrotic components. The majority of the tumors did not contain calcifications (5/6); displayed an ill-defined margin (4/6); were centered in the medulla; extended into the renal pelvis or peripelvic tissues (6/6); and did not exhibit a fibrous capsule. Localized caliectasis was observed in 3 of the 6 cases. The attenuation of the solid region of the RMC on unenhanced $\mathrm{CT}$ was equal to that of the renal cortex or medulla $(42.3 \pm 2.7$ vs. $40.7 \pm 3.6$ and 41.2 \pm 3.9 Hounsfield units, respectively; $P>0.05$ ) while, on enhanced CT, the enhancement of the tumor was lower than that of the normal renal cortex and medulla during all phases (cortical phase, $52.6 \pm 4.8$ vs. $199.5 \pm 9.7$ and $72.7 \pm 6.4$; medullary phase, $58.6 \pm 5.7$ vs. $184.6 \pm 10.8$ and $93.5 \pm 7.8$; delayed phase, $56.8 \pm 6.1$ vs. $175.7 \pm 8.5$ and $96.5 \pm 7.9$, respectively; $\mathrm{P}<0.05$ ).
\end{abstract}

Correspondence to: Dr Dairong Cao, Department of Radiology, First Affiliated Hospital of Fujian Medical University, 20 Cha-Zhong Road, Fuzhou, Fujian 350005, P.R. China

E-mail: shizhenshan0206@163.com

*Contributed equally

Key words: kidney neoplasm, renal medullary carcinoma, multislice computed tomography
In conclusion, $\mathrm{RMC}$ tends to be an infiltrative, ill-defined heterogeneous mass with intratumoral necrosis, which arises from the renal medulla, and displays lower enhancement than the renal cortex and medulla during all phases on enhanced CT. Despite its rarity in adults, RMC should be included in a differential diagnosis when $\mathrm{CT}$ imaging reveals these features.

\section{Introduction}

Renal medullary carcinoma (RMC) is a rare subtype of renal cell carcinoma that most commonly occurs in adolescents and young adults with sickle cell (SC) hemoglobinopathies (1). According to the World Health Organization (WHO) classification of renal tumors, RMC is a distinct entity with unique biological behavior and distinctive pathological and morphogenetic characteristics $(2,3)$. The most common symptoms of RMC are hematuria and flank or abdominal pain, which may lead to a misdiagnosis of urinary tract infection or renal abscess in certain patients, prior to a neoplasm being suspected (1).

A number of cases of RMC in non-African Americans without sickle cell anemia or sickle cell trait were reported within a series of 33 highly aggressive RMC first described in 1995 (1). The histopathological features of RMC include epithelial cells with reticular, adenoid cystic plasia, and prominent inflammation (4).

At present, the prognosis for patients with $\mathrm{RMC}$ remains very poor, since $95 \%$ of cases present at an advanced stage at the time of diagnosis and the tumor is resistant to chemotherapy in addition to biological therapy (4-6). A mean survival of 19 weeks from the time of initial diagnosis of RMC was reported by Simpson et al (7). Surgery of radical nephrectomy without metastatic disease appears to prolong survival of the patients $(8,9)$.

Pathologically, RMC arises from the renal medulla, grows rapidly in an infiltrative pattern, and invades the renal sinuses (10). Previous studies on RMC have documented the pathological and clinical features of this rare form of renal carcinoma (11). However, there are limited studies on RMC focusing on computed tomography (CT) imaging findings $(10,12)$. Patients with RMC present a poor prognosis, and nearly all patients succumb to the disease within several months following diagnosis. Therefore, an accurate diagnosis of RMC is important, since an early diagnosis may improve the prognosis of these patients. Therefore, the aim of the 
Table I. Clinical features of 6 Han Chinese patients with renal medullary carcinoma.

\begin{tabular}{lclcl}
\hline Patient no. & Age (years) & Gender & Hemoglobin status & \multicolumn{1}{c}{ Presentation } \\
\hline 1 & 72 & Female & Sickle cell trait & Renal mass \\
2 & 38 & Male & Unknown & Flank pain, hematuria \\
3 & 57 & Male & Unknown & Left flank pain, suspected renal calculus \\
4 & 56 & Female & Unknown & Abdominal pain \\
5 & 58 & Female & Unknown & Renal mass, fever, back pain \\
6 & 22 & Male & Unknown & Abdominal mass \\
\hline
\end{tabular}

Table II. Antibodies used for immunohistochemical analysis.

\begin{tabular}{lccccc}
\hline Antibody & Clone & Dilution & Manufacturer & Catalog no. & Host Target species \\
\hline CAM5.2 & CAM5.2 & prediluted & Thermo Fisher Scientific, Fremont, CA, USA & ZM-0316 & Mouse/Human \\
EMA & E29 & $1: 200$ & Thermo Fisher Scientific, Fremont, CA, USA & Kit-0011-2 & Mouse/Human \\
CK(H) & 34BE-12 & $1: 400$ & Thermo Fisher Scientific, Fremont, CA, USA & MAB-0052 & Mouse/Human \\
P504S & 13H4 & $1: 100$ & Zeta, Sierra Madre, CA, USA & ZA0227 & Rabbit/Human \\
Cytokeratin & AE1/AE3 & $1: 200$ & Dako, Glostrup, Denmark & Kit-0009-2 & Mouse/Human \\
\hline
\end{tabular}

present study was to investigate the $\mathrm{CT}$ imaging findings in 6 cases of RMC.

\section{Patients and methods}

Patients. An institutional review board exemption and a waiver of the requirement for written informed consent from the patients to perform the present retrospective study were obtained from the First Affiliated Hospital of Fujian Medical University (Fuzhou, China). A search in the pathology records and the picture archiving and communication system of the hospital identified 6 patients with RMC, who were hospitalized at the First Affiliated Hospital of Fujian Medical University between 2003 and 2014. Details of the patients, including age, gender, ethnicity and clinical symptoms, were recorded, in addition to characteristics of the tumor, including size, location (right or left), surgery or biopsy confirmation, and presence of metastasis, necrosis and/or hemorrhage, pyelocaliectasis, vascular invasion and SC trait.

Multi-slice CT examinations. All examinations were performed on multi-slice CT (MSCT) scanners (Aquilion 16 and Aquilion ONE; Toshiba Medical Systems Corporation, Otawara-shi, Japan), using the following abdominal scanning parameters: i) Detector collimation, $16.0 \times 0.5 \mathrm{~mm}(\mathrm{n}=4)$ or $320.0 \times 0.5 \mathrm{~mm}(\mathrm{n}=2)$; ii) gantry rotation time, $0.35-0.50 \mathrm{sec}$; iii) pitch, 1.0-1.4; iv) tube voltage, $120 \mathrm{kV}$; and v) abdominal reference tube current, 60-120 mA. All images were reconstructed from the contrast-enhanced MSCT scans with a slice thickness of $0.75-1.00 \mathrm{~mm}$ and reconstruction increments of $0.5 \mathrm{~mm}$.

For contrast-enhanced CT scanning, an 80-100-ml bolus of iopromide (300 mg/ml; Bayer HealthCare Pharmaceuticals, Berlin, Germany) was administered at a rate of $4-6 \mathrm{ml} / \mathrm{sec}$ via injection into an antecubital vein, followed by injection of
$40 \mathrm{ml}$ saline solution. The enhanced CT scans were initiated at 20-25 sec following injection for the arterial (cortical) phase; after 65-75 sec for the cortico-medullary (medullary) phase; and after 270-300 sec for the excretory (delayed) phase. In all cases where an initial non-contrast CT scan was available, the degree and pattern of enhancement of the tumor were determined in the nephrographic phase.

Pathological examination. Evaluation of gross specimens was conducted to assess their shape; presence of necrotic components; formation of fibrous capsule; and invasion into the renal pelvis, calyx, ureter, renal vein or inferior vena cava. Light microscopy was used to evaluate pathological specimens, using an XP-201 polarizing microscope (Nanjing Jiangnan Novel Optics Co.,Ltd., Nanjing, China), and an immunohistochemical analysis was also conducted. The tissue was obtained from the surgical resection or biopsy specimens in six cases. All tumors were fixed in neutral buffered formalin and all were paraffin embedded. Four-micron-thick sections of paraffin-embedded materials were cut, deparaffinized in xylene and rehydrated in descending dilution of ethanol. The sections were subjected to heat-induced epitope antigen retrieval using an electric pressue cooker set at $120^{\circ} \mathrm{C}$ for $5 \mathrm{~min}$. For CAM5.2, enzyme treatment (IP enzyme [ventana,Tucson,AZ,USA]) was used in addition to the heat-induced epitope antigen retrieval. The tissue was pretreated with $3 \%$ hydrogen peroxidase for 10 min to block endogenous peroxidase activity. The sections were incubated in $10 \%$ normal goat serum in PBS for 10 min to block nonspecific binding. The sections were washed three times for 5 min with TBS between incubation steps. The sections were incubated with the primary antibodies listed in Table II for $60 \mathrm{~min}$. The sections were then washed as before and incubated with the secondary antibody, RealTM EnVision (K5007; Dako Denmark) for $30 \mathrm{~min}$. Real TM DAB+Chromogen (Dako Denmark) was used as a chromogen for antigen localization. 
A
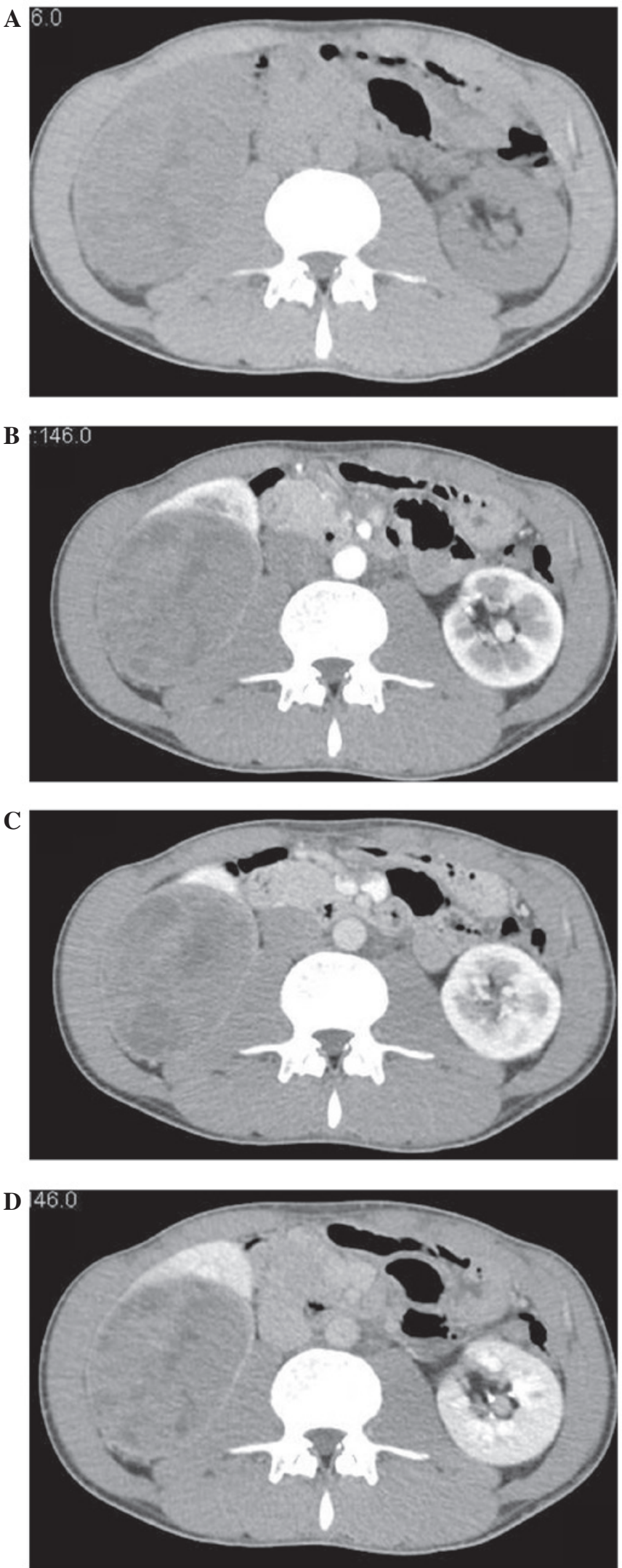

Figure 1. A 38-year-old male with renal medullary carcinoma in the right kidney. (A) Unenhanced CT scan revealed a well-defined heterogeneous mass, presenting an attenuation of the solid part of $38 \mathrm{HU}$. (B) During the cortical phase, contrast-enhanced CT scan indicated a mild enhancement of the tumor $(52 \mathrm{HU})$, which was lower than that of the renal cortex and medulla. In addition, a clear boundary was observed. (C) The attenuation of the tumor slightly increased during the medullary phase ( $58 \mathrm{HU})$. (D) During the delayed phase, the attenuation of the tumor decreased to $56 \mathrm{HU}$. CT, computed tomography; HU, Hounsfield units.
Table III. Attenuation of the renal tumors on dynamic contrast-enhanced computed tomography scan, compared with normal renal cortex and medulla.

\begin{tabular}{lccc}
\hline & \multicolumn{3}{c}{ Attenuation, Hounsfield units } \\
\cline { 2 - 4 } Phase & $\begin{array}{c}\text { Renal medullary } \\
\text { carcinoma }\end{array}$ & $\begin{array}{c}\text { Normal renal } \\
\text { cortex }\end{array}$ & $\begin{array}{c}\text { Normal renal } \\
\text { medulla }\end{array}$ \\
\hline Cortical & $52.6 \pm 4.8$ & $199.5 \pm 9.7$ & $72.7 \pm 6.4$ \\
Medullary & $58.6 \pm 5.7$ & $184.6 \pm 10.8$ & $93.5 \pm 7.8$ \\
Delayed & $56.8 \pm 6.1$ & $175.7 \pm 8.5$ & $96.5 \pm 7.9$ \\
\hline
\end{tabular}

Data is presented as the mean \pm standard deviation.

Slides were exposed to diaminobenzidine for $5 \mathrm{~min}$. After immunostaining, the sections were counterstained with hematoxylin, coverslipped and sealed. PBS was used as a negative control for the primary antibody for each group. All renal tumors were confirmed to be RMC based upon the histological examination and immunohistochemical findings $(1,13)$.

Image analysis. The imaging characteristics of the tumors were retrospectively evaluated by two genitourinary radiologists in consensus. The following parameters were evaluated in the tumors: Location; size; presence of calcification, cystic or necrotic components; and attenuation on unenhanced CT scan. The degree of enhancement of the tumors on enhanced CT was assessed during the aforementioned 3 phases, and the results were expressed in Hounsfield units (HUs). The presence of a capsule, hydronephrosis, perinephric stranding, vascular and renal tissue invasion, and metastases to retroperitoneal lymph node or other locations, was also documented.

On non-contrast enhanced CT, tumors were considered 'isodense' if their density in HU was equal to that of the renal parenchyma; 'high' if $>30 \mathrm{HU}$; 'mildly high' if $>10 \mathrm{HU}$; and 'low' if $<10 \mathrm{HU}$, compared with the contralateral normal renal parenchyma. The tumors were considered to be solid or cystic masses based on the predominance. Tumor location was categorized as medullary, cortical or exophytic based on the association of the tumor with the renal parenchyma, and perinephric or renal sinus fat. Thus, a medullary tumor, in which a component extended into the renal pelvis, was considered to possess a medullary location. Similarly, any tumor that was limited to the confines of the renal contour was considered to have a cortical location; and an exophytic location was assigned to any tumor extending beyond the renal contour. The absence or presence of a tumor boundary was assessed on the delayed phase of CT as a poorly or clearly defined margin.

The attenuation of the tumor and the normal renal medulla and cortex of the contralateral kidney were measured during the 3 enhanced phases of CT. Intratumoral calcifications and cystic components were excluded from the measured tumor area, which was situated at the center of the mass and was defined as the region of interest (ROI). In each phase, each $10-\mathrm{mm}$ area within the ROI was measured 4 times, and the mean value was calculated. The pattern of enhancement of the tumor was defined as heterogeneous or homogeneous, and its 


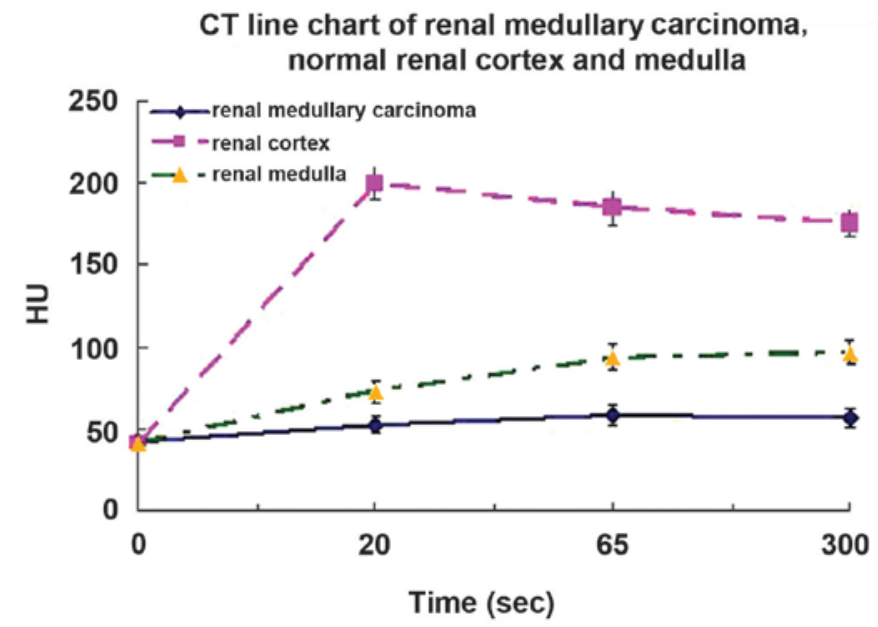

Figure 2. CT line chart of renal medullary carcinoma, normal renal cortex and medulla during unenhanced CT and the cortical, medullary and delayed phases of enhanced CT (n=6). CT, computed tomography; HU, Hounsfield units.

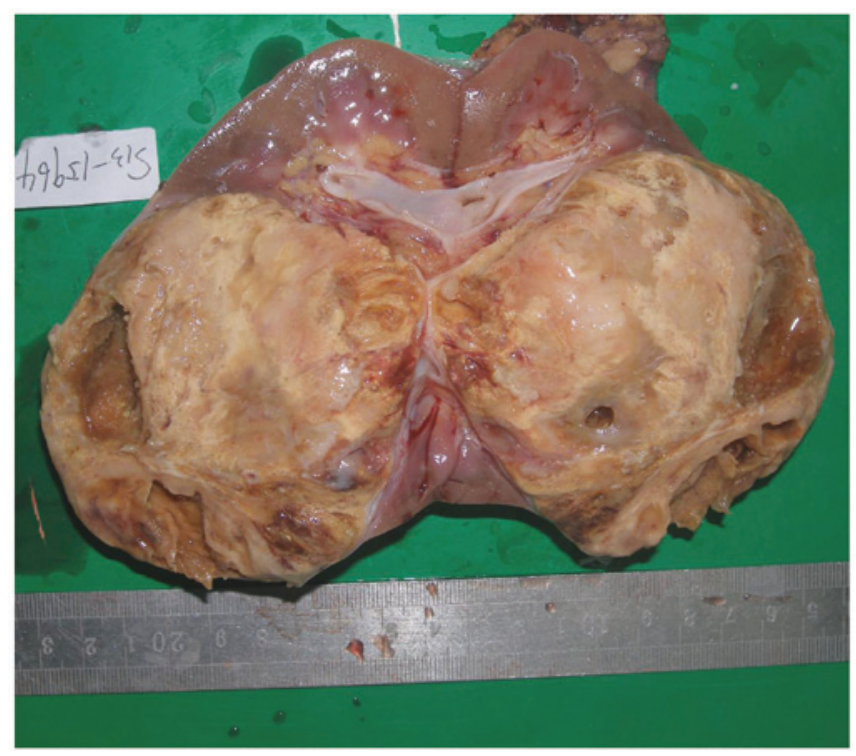

Figure 3. A fairly well-circumscribed yellow-white tumor located in the middle and low pole of the right kidney. Ruler indicates measurement in $\mathrm{cm}$.



Figure 4. The most common histological feature of renal medullary carcinoma is a cribriform architecture and stromal desmoplasia. degree of enhancement was based on the HU attenuation of the tumor, renal cortex and medulla.

Statistical analysis. Statistical analyses were performed with SPSS statistical software, version 3.0 (SPSS Inc., Chicago, USA). The numerical data were presented as the mean \pm standard deviation. One-way analysis of variance was used to compare the evaluated characteristics. $\mathrm{P}<0.05$ was considered to indicate a statistically significant difference.

\section{Results}

Patient characteristics. The present study included 6 patients with RMC (3 females and 3 males), whose clinical data are presented in Table I. The mean age of the patients at the time of diagnosis was 50.5 years (range, 22-72 years). Tumors were located in the right kidney in 3 cases, and the left kidney in the remaining 3 cases. The diameter of the tumors ranged from 2.90 to $10.50 \mathrm{~cm}$ (mean diameter, $7.48 \pm 3.25 \mathrm{~cm}$ ), and tumor shape was observed to be oval in 4 cases, and irregular in 2 . Of the 6 patients, 2 presented with retroperitoneal lymph node metastasis, and 3 with hydronephrosis. According to hemoglobin analyses, SC hemoglobinopathy was present in only 1 case, whereas the presence of SC trait was unknown for the remaining 5 cases, prior to detection of the tumor. There was no bias towards any particular location of the tumors or gender of the patients among the 6 cases included in the study.

Mass position. All RMCs were located in the medulla and invaded the renal pelvis. The tumors extended to the renal cortex in 4 cases, and to the perirenal tissue in 1 case.

Mass appearance. In all 6 cases, the tumor mass was predominantly solid and heterogeneous, and presented cystic or necrotic components. Punctate calcifications were detected in 1 case. A poorly defined margin of the RMC was observed in 4 cases on the delayed phase of CT, whereas the margin of the remaining 2 cases appeared well-defined. No fibrous capsule was present in any of the 6 cases. In 1 case, the tumor had spread into the retroperitoneal soft tissue, invaded the left renal artery, and developed regional lymph node metastasis.

Attenuation of normal kidney and tumor on unenhanced CT. The CT attenuation of the RMC was equal to that of the normal renal cortex and medulla $(42.3 \pm 2.7$ vs. $40.7 \pm 3.6$ and 41.2 $\pm 3.9 \mathrm{HU}$, respectively; $\mathrm{P}>0.05$ ). On unenhanced $\mathrm{CT}$, the attenuation of the solid part of the RMC was isodense, equal to that of the normal renal parenchyma, in all 6 cases (Fig. 1).

Degree and pattern of CT enhancement. On dynamic contrast-enhanced CT scan, the attenuation of RMC was markedly lower than that of the normal renal cortex and medulla during all enhanced phases $(\mathrm{P}<0.05, \mathrm{n}=6$; Table III and Fig. 2).

Surgical/gross observation and follow-up. All 6 patients with RMC underwent surgery. In 4 cases, the masses were oval, and in 2 cases, the masses were irregular in shape and exhibited regional lymph node metastasis. The tumor masses were firm or rubbery with white-to-grey color, and occupied the medulla (Fig. 3). All 6 tumors had invaded into the renal pelvis or calyx. 
Despite the total nephrectomy performed, the patients presented a poor outcome. Consequently, chemotherapy and/or immunotherapy was administered post-surgically in 5 cases: One case received sunitinib at a dose of $50 \mathrm{mg} / \mathrm{day}$, administered in one 6-week cycle of daily oral therapy for 4 weeks, followed by 2 weeks off. After the cycle, abdominal CT images revealed an enlargement of the previously observed retroperitoneal lymph nodes. The patient was then treated with two 7-week cycles of high-dose-intensity MVAC (methotrexate, vinblastine, doxorubicin, and cisplatin), which was initially described by Sternberg et al $(14,15)$. Doses of $30 \mathrm{mg} /$ $\mathrm{m}^{2}$ methotrexate and $3.0 \mathrm{mg} / \mathrm{m}^{2}$ vinblastine were administered by intravenous infusion on days 1 and 15 . Doses of $30 \mathrm{mg} /$ $\mathrm{m}^{2}$ doxorubicin and $70 \mathrm{mg} / \mathrm{m}^{2}$ cisplatin were administered by intravenous infusion on days 2 and 16 of 28-day cycles. The other cases were treated with 1-3 cycles of high-dose-intensity MVAC. By contrast, 1 case diagnosed with increasing metastatic burden did not receive adjuvant therapy. The clinical condition of the patients rapidly deteriorated following diagnosis of RMC. The median survival time of the 6 cases from the time of diagnosis was 11 weeks.

Pathological findings. All 6 tumors displayed similar microscopic characteristics, including a cribriform architecture and stromal desmoplasia (Fig. 4). The reticular pattern of growth consisted of tumor cell aggregates forming spaces of different sizes. An adenoid-cystic pattern of growth was further identified in 5 tumors. Necrosis was noted in all cases. Immunohistochemical studies confirmed homogeneous expression of low molecular weight cytokeratin CAM 5.2, and co-expression of vimentin and epithelial membrane antigen in all tumors.

\section{Discussion}

According to the 2004 WHO classification of renal tumors, RMC is a rare subtype of renal cell carcinoma that was first described as the 'seventh sickle cell nephropathy' $(1,16)$. The age range of the patients in the present study was 22-72 years. This population is older than that reported by Davis et al (1) (range, 11-39 years), possibly due to the fact that the patients of the present study belonged to the Han Chinese population, in which SC hemoglobinopathies are rare. Accordingly, only 1 of the 6 cases in the present study was diagnosed with SC hemoglobinopathy by hemoglobin analysis.

The most significant clinical challenge presented by RMC is that chemo-, immuno- and radiotherapy have all been unsuccessful for the treatment of RMC, based on previous studies $(4,5,17-22)$. Therefore, an early diagnosis may improve the survival rates of patients with RMC. Although RMC has been relatively well described in previous pathological studies (23-25), the imaging data published in these studies are limited, particularly regarding histopathological examinations. In routine clinical work, a correct imaging diagnosis of RMC is difficult, which is mostly likely due to the low morbidity and level of awareness of this disease (26). The data of the present study suggest that the following imaging characteristics may aid in identifying RMC accurately: RMC tends to poorly circumscribed, solitary and heterogeneous mass, which arises from the renal medulla, and presents lower enhancement compared with that of the cortex and medulla in all phases of CT. In addition, RMC exhibits an infiltrative appearance, and usually extends into the renal pelvis and peripelvic tissues.

Histopathologically, RMC is known to arise from the renal medulla, as reported in previous pathological studies $(23,24)$. Since other renal tumors, including parts of clear cell renal cell carcinoma, transitional cell carcinoma, rhabdoid tumor and renal lymphoma may also involve the medulla, differentiating RMC from such other tumors may be challenging based solely on the location of the tumor (26). However, a number of imaging and clinical features may facilitate this differentiation: The majority of cortical clear cell renal cell carcinomas $(\sim 94 \%)$ have an expansible appearance, and display exophytic growth that disrupts the reniform contour (27). Their degree of enhancement is usually higher than that of the normal renal cortex (28). These findings suggest that distinguishing between RMCs and other renal tumors with a rich blood supply based on their different enhancement on CT may be feasible. Transitional cell carcinomas arise from the collecting system, however, gross hematuria and hydronephrosis are common at the initial staging (29). Rhabdoid tumor of the kidney usually occurs in patients under 3 years of age, although its appearance is similar to that of RMC $(30,31)$. Renal lymphoma is usually bilateral and multifocal, and mostly occurs in patients with non-Hodgkin's lymphoma, although it is evident only in $\sim 5 \%$ of patients at presentation $(32,33)$.

The results of the present study indicate that RMCs are heterogeneous masses with an isodense parenchyma on unenhanced CT scan, which is considered to be due to their stromal desmoplasia. On pathology and surgery, satellite lesions in the renal cortex and intratumoral hemorrhage were observed in 5 cases and necrosis was observed in all the cases. This combination of traits differs from that of solid tumors that exhibit high attenuation, such as clear cell renal cell and papillary carcinomas, oncocytomas and angiomyolipomas with minimal fat (34).

In the present study, the enhancement of RMCs on CT was lower than that of the renal cortex and medulla during all enhanced phases of CT, which was hypothesized to be due to the rare hypovascular features of this type of tumor. This pattern of enhancement is atypical of tumors with a rich blood supply, including renal angiomas, renal angiomyolipomas with minimal fat and clear cell renal cell carcinomas, whose degree of enhancement is normally higher than that of the normal renal cortex (35). Therefore, these findings support the concept that distinguishing between RMCs and renal tumors with a rich blood supply based on their different enhancement on CT may be relatively easy.

Due to their similar characteristics, RMC was often misclassified in the past as an aggressive form of collecting duct carcinoma, prior to being recognized as a separate entity. Both RMCs and collecting duct carcinomas present an infiltrative pattern, are biologically aggressive, arise from the medulla, and are considered to derive from proliferating cells of the collecting duct epithelium, although collecting duct carcinoma is not associated with hemoglobinopathies $(23,36,37)$. Therefore, it is often difficult to distinguish between these 2 types of tumors by using exclusively dynamic contrast-enhanced CT imaging. 
In conclusion, the presence of an infiltrative, heterogeneous mass with necrosis, which arises from the renal medulla and displays lower enhancement than that of the renal cortex and medulla during all phases of CT, suggests the diagnosis of RMC.

\section{Acknowledgements}

The present study was supported by a grant from the scientific research programs of Fujian provincial Health and Family Planning Commission for young scholars (2014-01-45). The authors are grateful to Dr Xianying Zhen and Dr Kaige Wu, for their advice and support at various stages of the project.

\section{References}

1. Davis CJ Jr, Mostofi FK and Sesterhenn IA: Renal medullary carcinoma. The seventh sickle cell nephropathy. Am J Surg Pathol 19: 1-11, 1995.

2. Skolarus TA, Serrano MF, Berger DA, Bullock TL, Yan Y, Humphrey PA and Kibel AS: The distribution of histological subtypes of renal tumors by decade of life using the $2004 \mathrm{WHO}$ classification. J Urol 179: 433-444, 2008.

3. Lopez-Beltran A, Scarpelli M, Montironi R and Kirkali Z: 2004 WHO classification of the renal tumors of the adults. Eur Urol 49: 798-805, 2006.

4. Schaeffer EM, Guzzo TJ, Furge KA, Netto G, Westphal M, Dykema K, Yang X, Zhou M, Teh BT and Pavlovich CP: Renal medullary carcinoma: Molecular, pathological and clinical evidence for treatment with topoisomerase-inhibiting therapy. BJU Int 106: 62-65, 2010.

5. Hakimi AA, Koi PT, Milhoua PM, Blitman NM, Li M, Hugec V, Dutcher JP and Ghavamian R: Renal medullary carcinoma: The Bronx experience. Urol 70: 878-882, 2007.

6. Sathyamoorthy K, Teo A and Atallah M: Renal medullary carcinoma in a patient with sickle-cell disease. Nat Clin Pract Urol 3: 279-283, 2006.

7. Simpson L, He X, Pins M, Huang X, Campbell SC, Yang XJ, Perlman EJ and Bergan RC: Renal medullary carcinoma and ABL gene amplification. J Urol 173: 1883-1888, 2005.

8. Khan A, Thomas N, Costello B, Jobling L, deKretser D, Broadfield $\mathrm{E}$ and $\mathrm{O}$ 'Shea $\mathrm{S}$ : Renal medullary carcinoma: Sonographic, computed tomography, magnetic resonance and angiographic findings. Eur J Radiol 35: 1-7, 2000.

9. Selby DM, Simon C, Foley JP, Thompson IM and Baddour RT: Renal medullary carcinoma: Can early diagnosis lead to long-term survival? J Urol 163: 1238, 2000.

10. Blitman NM, Berkenblit RG, Rozenblit AM and Levin TL: Renal medullary carcinoma: CT and MRI features. AJR Am J Roentgenol 185: 268-272, 2005.

11. Swartz MA, Karth J, Schneider DT, Rodriguez R, Beckwith JB and Perlman EJ: Renal medullary carcinoma: Clinical, pathologic, immunohistochemical, and genetic analysis with pathogenetic implications. Urology 60: 1083-1089, 2002.

12. Neville A and Hatem SF: Renal medullary carcinoma: Unsuspected diagnosis at stone protocol CT. Emerg Radiol 14: 245-247, 2007.

13. Steele EL and MacLennan GT: Renal medullary carcinoma. J Urol 174: 1449, 2005.

14. Sternberg CN, de Mulder P, Schornagel JH, Theodore C, Fossa $\mathrm{SD}$, van Oosterom AT, Witjes JA, Spina M, van Groeningen CJ, Duclos B, et al; EORTC Genito-Urinary Cancer Group: Seven year update of an EORTC phase III trial of high-dose intensity M-VAC chemotherapy and G-CSF versus classic M-VAC in advanced urothelial tract tumours. Eur J Cancer 42: 50-54, 2006.

15. Sternberg CN, de Mulder PH, Schornagel JH, Théodore C, Fossa SD, van Oosterom AT, Witjes F, Spina M, van Groeningen CJ, de Balincourt C and Collette L; European Organization for Research and Treatment of Cancer Genitourinary Tract Cancer Cooperative Group: Randomized phase III trial of high-doseintensity methotrexate, vinblastine, doxorubicin, and cisplatin (MVAC) chemotherapy and recombinant human granulocyte colony-stimulating factor versus classic MVAC in advanced urothelial tract tumors: European Organization for Research and Treatment of Cancer Protocol no. 30924. J Clin Oncol 19: 2638-2646, 2001.
16. Lopez-Beltran A, Scarpelli M, Montironi R and Kirkali Z: 2004 WHO classification of the renal tumors of the adults. Eur Urol 49: 798-805, 2006

17. Strouse JJ, Spevak M, Mack AK, Arceci RJ, Small D and Loeb DM: Significant responses to platinum-based chemotherapy in renal medullary carcinoma. Pediatr Blood Cancer 44: 407-411, 2005.

18. Walsh A, Kelly DR, Vaid YN, Hilliard LM and Friedman GK: Complete response to carboplatin, gemcitabine, and paclitaxel in a patient with advanced metastatic renal medullary carcinoma. Pediatr Blood Cancer 55: 1217-1220, 2010.

19. Ronnen EA, Kondagunta GV and Motzer RJ: Medullary renal cell carcinoma and response to therapy with bortezomib. J Clin Oncol 24: e14, 2006.

20. Maroja Silvino MC, Venchiarutti Moniz CM, Munhoz Piotto GH, Siqueira S, Galapo Kann A and Dzik C: Renal medullary carcinoma response to chemotherapy: a referral center experience in Brazil. Rare Tumors 5: e44, 2013.

21. Stahlschmidt J, Cullinane C, Roberts P and Picton SV: Renal medullary carcinoma: Prolonged remission with chemotherapy, immunohistochemical characterisation and evidence of bcr/abl rearrangement. Med Pediatr Oncol 33: 551-557, 1999.

22. Walsh AM, Fiveash JB, Reddy AT and Friedman GK: Response to radiation in renal medullary carcinoma. Rare Tumors 3: e32, 2011.

23. Amin MB, Smith SC, Agaimy A, Argani P, Compérat EM, Delahunt B, Epstein JI, Eble JN, Grignon DJ, Hartmann A, et al: Collecting duct carcinoma versus renal medullary carcinoma: An appeal for nosologic and biological clarity. Am J Surg Pathol 38: 871-874, 2014.

24. Liu Q, Galli S, Srinivasan R, Linehan WM, Tsokos M and Merino MJ: Renal medullary carcinoma: Molecular, immunohistochemistry, and morphologic correlation. Am J Surg Pathol 37: 368-374, 2013.

25. Prasad SR, Humphrey PA, Catena JR, Narra VR, Srigley JR, Cortez AD, Dalrymple NC and Chintapalli KN: Common and uncommon histologic subtypes of renal cell carcinoma: Imaging spectrum with pathologic correlation. Radiographics 26: 1795-1810, 2006.

26. Blitman NM, Berkenblit RG, Rozenblit AM and Levin TL: Renal medullary carcinoma: CT and MRI features. AJR Am J Roentgenol 185: 268-272, 2005.

27. Yoshimitsu K, Irie H, Tajima T, Nishie A, Asayama Y, Hirakawa M, Nakayama T, Kakihara D and Honda H: MR imaging of renal cell carcinoma: Its role in determining cell type. Radiat Med 22: 371-376, 2004.

28. Zhu Q, Zhu W, Wang Z and Wu J: Clinical and CT imaging features of mucinous tubular and spindle cell carcinoma. Chin Med J (Engl) 127: 1278-1283, 2014.

29. Strobel SL, Jasper WS, Gogate SA and Sharma HM: Primary carcinoma of the renal pelvis and ureter. Evaluation of clinical and pathologic features. Arch Pathol Lab Med 108: 697-700, 1984.

30. Farmakis SG and Siegel MJ: Rhabdoid tumor: An aggressive renal medullary tumor of childhood. J Comput Assist Tomogr 39: 44-46, 2015.

31. Zhang Z, Chen J, Zhou J, Liu Y, Feng Z, Tang L and Jin Y: Clinicopathological study and diagnosis of rhabdoid tumor of kidney combined with metanephric adenoma. Chin Med J (Engl) 127: 4290-4291, 2014.

32. Ganeshan D, Iyer R, Devine C, Bhosale P and Paulson E: Imaging of primary and secondary renal lymphoma. AJR Am J Roentgenol 201: 712-719, 2013.

33. Navas Martínez MC, Molina Escudero R, Soto Delgado M, Jiménez Romero ME, Jiménez Jiménez J: Bilateral primary renal lymphoma: Case report and bibliographic. Arch Esp Urol 64: 904-907, 2011.

34. Swartz MA, Karth J, Schneider DT, Rodriguez R, Beckwith JB and Perlman EJ: Renal medullary carcinoma: clinical, pathologic, immunohistochemical, and genetic analysis with pathogenetic implications. Urol 60: 1083-1089, 2002.

35. Hindman N, Ngo L, Genega EM, Melamed J, Wei J, Braza JM, Rofsky NM and Pedrosa I: Angiomyolipoma with minimal fat: Can it be differentiated from clear cell renal cell carcinoma by using standard MR techniques? Radiology 265: 468-477, 2012.

36. Soto Delgdo M, Pedrero Márquez G, Arroyo Maestre JM, and Beardo Villar P: Collecting duct carcinoma of the kidney. A contribution of 4 new cases. Arch Esp Urol 67: 714-717, 2014.

37. Kwon KA, Oh SY, Kim HY, Kim HS, Lee HY, Kim TM, Lim HY, Lee NR, Lee HJ, Hong SH and Rha SY: Clinical features and treatment of collecting duct carcinoma of the kidney from the Korean cancer study group genitourinary and gynecology cancer committee. Cancer Res Treat 46: 141-147, 2014. 